\title{
Disk of 2MASS 15491331-3539118 = GQ Lup C as seen by HST and WISE`
}

\author{
C. Lazzoni ${ }^{1,2}$, R. Gratton ${ }^{1}$, J. M. Alcalá ${ }^{3}$, S. Desidera ${ }^{1}$, A. Frasca ${ }^{4}$, C. F. Manara ${ }^{5}$, D. Mesa ${ }^{1}$, E. Rigliaco ${ }^{1}$, \\ A. Vigan ${ }^{6}$, and A. Zurlo ${ }^{7,8}$ \\ 1 INAF - Osservatorio Astronomico di Padova, Vicolo dell'Osservatorio 5, 35122 Padova, Italy \\ e-mail: cecilia.lazzoni@inaf.it \\ 2 Dipartimento di Fisica a Astronomia “G. Galilei”, Universita' di Padova, Via Marzolo, 8, 35121 Padova, Italy \\ 3 INAF - Osservatorio Astronomico di Capodimonte, Salita Moiariello 16, 80131 Napoli, Italy \\ ${ }^{4}$ INAF - Osservatorio Astronomico di Catania, Via Santa Sofia, 78, Catania, Italy \\ 5 European Southern Observatory, Karl-Schwarzschild-Strasse 2, 85748 Garching bei München, Germany \\ 6 Aix Marseille Université, CNRS, LAM (Laboratoire d'Astrophysique de Marseille) UMR 7326, 13388 Marseille, France \\ 7 Núcleo de Astronomía, Facultad de Ingeniería y Ciencias, Universidad Diego Portales, Av. Ejercito 441, Santiago, Chile \\ ${ }^{8}$ Escuela de Ingeniería Industrial, Facultad de Ingeniería y Ciencias, Universidad Diego Portales, Av. Ejercito 441, Santiago, Chile
}

Received 30 January 2020 / Accepted 1 March 2020

\section{ABSTRACT}

\begin{abstract}
Aims. Very recently, a second companion on a wider orbit has been discovered around GQ Lup. This is a low-mass accreting star that is partially obscured by a disk seen at high inclination. If detected, this disk may be compared to the known disk around the primary. Methods. We detected this disk on archive HST and WISE data.

Results. The extended spectral energy distribution provided by these data confirms the presence of accretion from H $\alpha$ emission and UV excess, and shows an IR excess attributable to a warm disk. In addition, we resolved the disk on the HST images. It is found to be roughly aligned with the disk of the primary. Both of them are roughly aligned with the Lupus I dust filament containing GQ Lup.
\end{abstract}

Key words. stars: individual: GQ Lup - stars: individual: 2MASS 15491331-3539118 - techniques: high angular resolution protoplanetary disks

\section{Introduction}

Alcalá et al. (2020; hereafter Paper I) recently found a probable second companion on a wider orbit (2MASS 154913313539118; projected separation $\sim 2400 \mathrm{au}$ ) to the very young star GQ Lup using Gaia DR2 data (Gaia Collaboration et al. 2018) that also has a closer brown dwarf (BD) companion (projected separation $\sim 100 \mathrm{au}$ ). Paper I found that GQ Lup C is accreting; the data strongly suggest that it may be surrounded by a disk seen nearly edge on that attenuates the stellar light, but may leave emission by an outflow unperturbed. However, the disk could not be detected from the spectral energy distribution (SED) considered in that paper because the SED only extends up to the $K$ band. The purpose of this paper is to find further evidence for this disk. We found that this can be obtained by extending the spectral range by considering observations acquired with the Wide-field Infrared Survey Explorer (WISE), and using high spatial resolution images such as those provided by the Hubble Space Telescope (HST). On the other side, GQ Lup C is too far from the primary to have been observed with the previous survey with the Atacama Large Millimeter/submillimeter Array (ALMA).

^ Based on observations made with the NASA/ESA Hubble Space Telescope, obtained from the data archive at the Space Telescope Science Institute. STScI is operated by the Association of Universities for Research in Astronomy, Inc. under NASA contract NAS 5-26555.

\section{Archival data}

\subsection{HST data}

Images of the region around GQ Lup in nine different bands were obtained with the HST Wide Field Camera 3 (WFC3; proposal 12507) at epoch 2012.15. These images include GQ Lup C. We retrieved them using the MAST archive interface ${ }^{1}$ in order to extend the SED to the UV and to exploit the high spatial resolution to possibly resolve the disk. We also retrieved HST NICMOS images, but GQ Lup C is outside the observed field.

\subsection{WISE data}

GQ Lup C is not listed in the WISE (Wright et al. 2010) point source catalog because it is not well resolved from the much brighter primary. In order to constrain the thermal IR emission from GQ Lup C, we then retrieved WISE images of the region around GQ Lup using the NASA-IRSA interface ${ }^{2}$. As mentioned above, the WISE point spread function (PSF) is very extended (full width at half-maximum, $F W H M \sim 10 \operatorname{arcsec}$, i.e., much larger than the size of the disk around $\mathrm{A}$ ), and $\mathrm{C}$ is much fainter than $\mathrm{A}$. To reduce the contamination by A, we simply subtracted from each image the same image rotated by 180 degree. The

\footnotetext{
https://archive.stsci.edu/hst/

2 https://irsa.ipac.caltech.edu/Missions/wise.html
} 

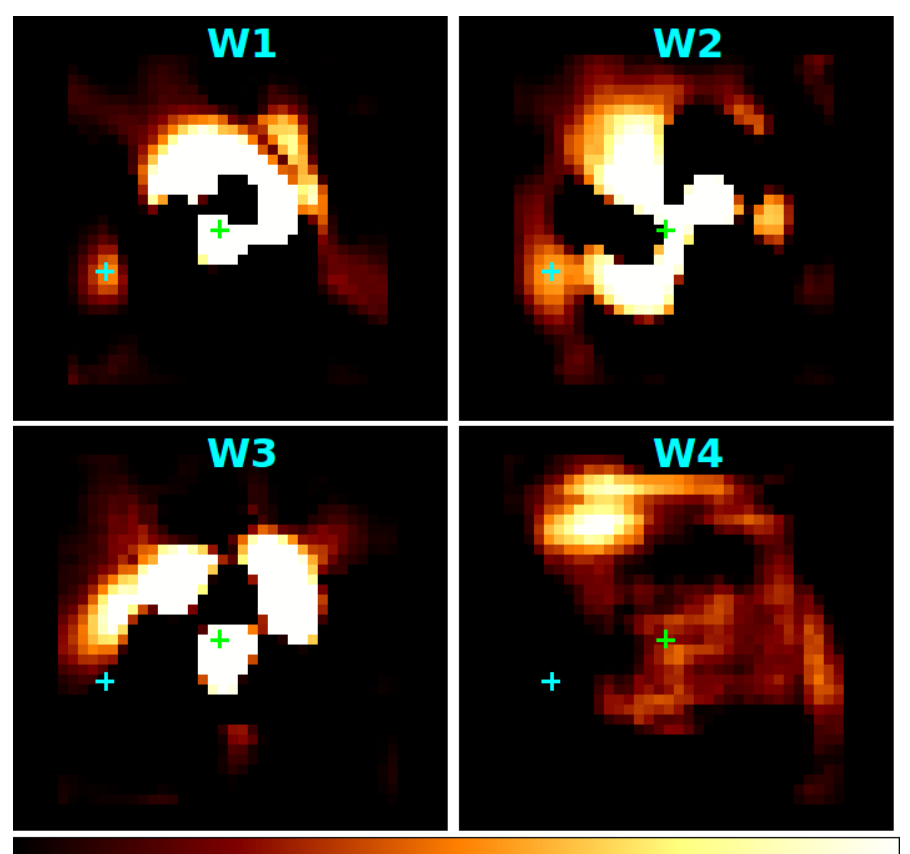

$\begin{array}{lllllllll}0.00050 & 0.00100 & 0.00150 & 0.00200 & 0.00250 & 0.00300 & 0.00350 & 0.00400 & 0.00450\end{array}$

Fig. 1. Images in the four different bands of WISE after subtracting the signal from GQ Lup A, as described in the text. The positions of GQ Lup $A$ and $C$ are marked by green and cyan crosses, respectively. In all these images, north is up and east to the left. GQ Lup C is detected in $\mathrm{W} 1$ and $\mathrm{W} 2$, but not at longer wavelengths.

exact position of the pivot of the rotation was obtained by minimizing the rms within a square region of $11 \times 11$ pixels centered on a first approximate position obtained by a Gaussian fitting routine. In this way, symmetric features on the PSF are suppressed $^{3}$. Figure 1 gives the result of this subtraction. There is a clear signal at the position of $\mathrm{C}$ in $\mathrm{W} 1$ and $\mathrm{W} 2$, while nothing is detected in W3 and W4. We used the nondetection in W3 to provide an upper limit to the emission by GQ Lup C, while we performed aperture photometry for $\mathrm{W} 1$ and $\mathrm{W} 2$.

\section{Spectral energy distribution}

In order to discuss the SED of GQ Lup C, we list in Table B.1 the fluxes over a wide spectral range (from 0.33 up to $12 \mu \mathrm{m}$ ) that we obtained combining HST, 2MASS (Skrutskie et al. 2006), and WISE data (Wright et al. 2010). The HST magnitudes were calibrated into flux using the recipes in the WFC3 handbook ${ }^{4}$.

Figure 2 shows a comparison between the SED of GQ Lup C and a BT-Settl model spectrum (Allard et al. 2012) with the parameters closest to those found in Paper I, that is, $T_{\text {eff }}=$ $3200 \mathrm{~K}$ and $\log g=3.5 \mathrm{dex}$. An extinction of $A_{V} \sim 1.0 \mathrm{mag}$, as found in Paper I, was also considered. The agreement is excellent in the spectral range between 0.4 and $2.2 \mu \mathrm{m}$. However, the HST data show evidence for strong excesses in the $\mathrm{H} \alpha$ narrow-band filter and in the UV, which are clear signs of mass accretion from the disk. By comparing the flux in the $F 656 N$ filter with that measured in broad-band filters and taking the band width into account, we obtained an equivalent width of $188 \pm 3 \AA$ for $\mathrm{H} \alpha$, that is, considerably stronger than the value of $100 \AA$ obtained from the X-shooter spectrum in Paper I. This argues for a strong

\footnotetext{
3 We also downloaded images of nearby objects, but their images look quite different and therefore cannot be used for a PSF subtraction.

4 https://hst-docs.stsci . edu/display/WFC3IHB
}

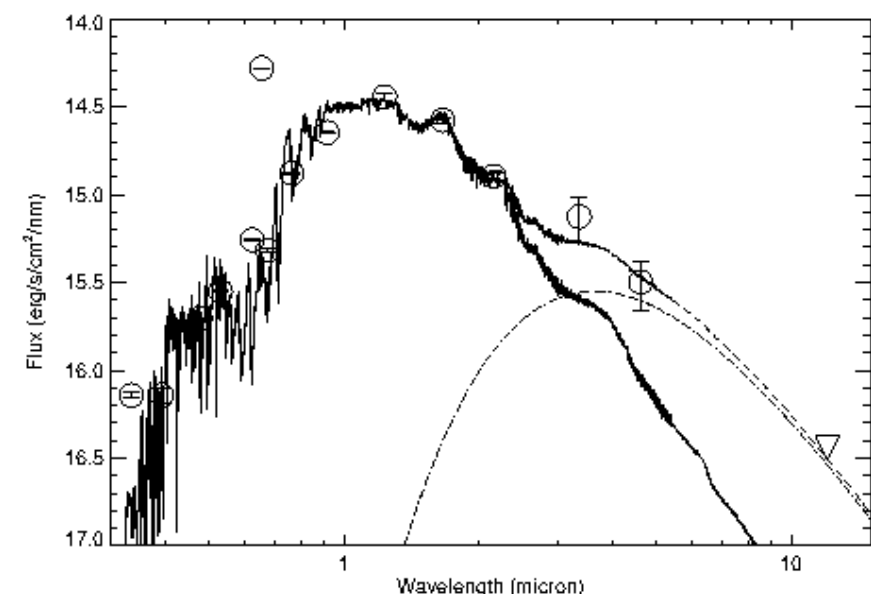

Fig. 2. Observed SED for GQ Lup C; circles are measures form HST, 2MASS, and WISE data; the down-pointing triangle is the upper limit in the W3 band. The solid line is the prediction for a BT-Settl model (Allard et al. 2012) of $3200 \mathrm{~K}$, with an absorption of $A_{V}=1.0 \mathrm{mag}$ (see Paper I), the dash-dotted line is a blackbody spectrum with a temperature of $800 \mathrm{~K}$, and the dashed line is the sum of the two. The discordant point is the HST-F656N H $\alpha$ band.

variability of the source; this has frequently been observed in accreting objects (see, e.g., Frasca et al. 2018, and references therein).

The SED also shows an IR excess at wavelengths longer than $3 \mu \mathrm{m}$, consistent with the detection of the warm part of the disk around GQ Lup C. This may be fit by a blackbody SED of $800 \pm 100 \mathrm{~K}$, a value that is constrained at the upper edge by the observed $K$ magnitude and at the lower edge by the non detection in W3. This is lower than the dust sublimation temperature, suggesting a real truncation of the disk. More specifically, this temperature is about one-fourth of the stellar temperature, suggesting that the disk is truncated at $R_{\mathrm{T}} \sim 15-20 R_{*}, R_{*}$ being the stellar radius. This may be compared with the value of $R_{\mathrm{T}} / R_{*}$ given by Eq. (2.2) of Bouvier et al. (2007): assuming $B=1 \mathrm{kG}$, $\dot{M}=3.3 \times 10^{-10} M_{\odot} \mathrm{yr}^{-1}, M_{*}=0.15 M_{\odot}$, and $R_{*}=0.87 R_{\odot}$ (Paper I), we obtain $R_{\mathrm{T}} / R *=12.3$. By integrating the fluxes from the model atmosphere and the blackbody representing the emission from the warm disk over all wavelengths, we obtain a value of $L_{\text {disk }}=L_{\text {bol }}-L_{\text {star }}=0.053 L_{\mathrm{star}}$, where $L_{\mathrm{bol}}$ is the total luminosity; this is of course a lower limit to the disk emission because we cannot exclude that there is also a cold component emitting at wavelengths longer than those considered here. Using Spitzer data to estimate $L_{\mathrm{bol}}$, Merín et al. (2008) obtained an average value of $L_{\text {disk }}=0.25 L_{\text {star }}$, but with considerable scatter, with values ranging from 0.01 to 1.5 . Accretors are commonly thought to have $L_{\text {disk }} / L_{\text {star }}>0.08$. GQ Lup C would then be at the lower edge of the distribution of this quantity.

\section{Disk images from HST}

One star (Gaia DR2 source 6011522757637551616) has a similar peak intensity and lies less than 2 arcsec from GQ Lup C (farther from GQ Lup A). This star can be used as a comparison source for HST images because it is placed at similar separation from the field center and is quite bright. A comparison of the flux of GQ Lup C with that of this comparison star confirms the clear detection of a strong $\mathrm{H} \alpha$ emission and UV excess for GQ Lup C. In addition and most interestingly, the GQ Lup C image appears to be fuzzier than the comparison star. While the peak intensity 

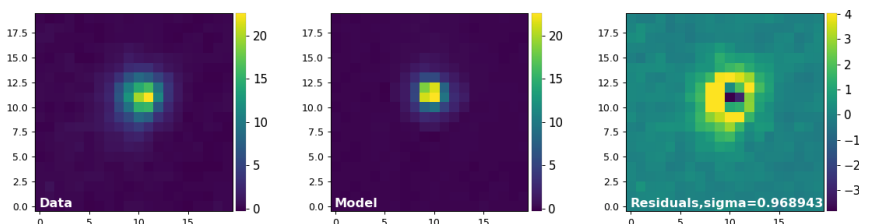

Fig. 3. Disk of GQ Lup C from the HST data after applying the PSF subtraction procedure. North is up and east to the left, and the scale is in pixel (one pixel is equal to 0.03962 arcsec).
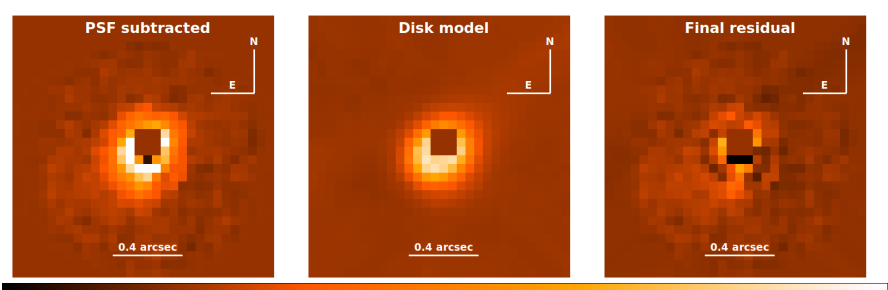

Fig. 4. Illustration of the method we used to estimate the disk parameters. Left panel: image after PSF subtraction. Central panel: model disk. Right panel: same as the left panel, but after subtracting the model disk.

is lower at most wavelengths, the FWHMs of the images are consistently higher (about 130 mas, compared to about 80 mas for the comparison object). This suggests that the disk around GQ Lup C is resolved from the stellar PSF in the HST images.

In order to image the disk from the HST data, we used a technique based on the PSF subtraction (see Appendix A for a more extended discussion). As a first step, we determined the position and the flux for GQ Lup C in each frame. Then we considered the PSF of the reference star and rescaled it to the flux we obtained for GQ Lup C. As a last step, we subtracted the reference PSF from our target star in its position and rotated the frame with respect to true north (see next section). The residuals are shown in the last panel of Fig. 3, and the first two images show GQ Lup C and the model PSF we used for the subtraction, respectively.

The PSF subtraction yields a well-resolved image of the disk of GQ Lup C. We consider here the image obtained by combining the four best bands (F850LP, F625W, F555W, and $F 775 \mathrm{~W}$ ); however, very similar results are obtained for the individual bands. After PSF subtraction, the excess signal appears to be approximately distributed in an ellipse that is aligned NW-SE. Its $\mathrm{E}$ side is brighter than the $\mathrm{W}$ side; the central empty region is likely an artifact of the procedure. This light distribution is probably produced by scattering of the stellar light by a flared circular disk that is seen at rather high inclination, with the near side less luminous because it is very close to the center of the stellar image and then subtracted by the procedure.

In order to determine the geometrical parameters of this disk, we assumed that the disk is a circular ring of radius $r$ seen at a given inclination $i$ and position angle $P A$, and convoluted it with the PSF of the nearby comparison star. We considered that the ring can be offset with respect to the center of the star along the minor axis of the projected ellipse, as seen in numerous cases of disks that are observed in scattered light (see, e.g., Ginski et al. 2016; Sissa et al. 2018). We then found the set of parameters that minimizes the rms of the residuals between the image obtained after the PSF subtraction and the model disk. Figure 4 illustrates the procedure. We repeated this procedure for the images obtained with the individual bands and assumed the mean value of the parameters as best estimate, and the standard deviation of the mean as its uncertainty. In this way, we obtained a radius of $r=2.22 \pm 0.14$ pixels (i.e., $88 \pm 6$ mas, or $13.2 \pm 0.8$ au at the distance of GQ Lup), an inclination $i=44 \pm 2$ degree, a position angle $P A=315 \pm 4$ degree, and an offset of $0.97 \pm 0.15$ pixel (i.e., $38 \pm 6$ mas) in the NE direction. The offset can be interpreted as due to flaring of the disk at an angle of about $26 \pm 7$ degree, the near side being SW.

For comparison, the disk of GQ Lup A has $P A=346 \pm$ 1 and $i=60.5 \pm 0.5$ degree, as derived from ALMA data (MacGregor et al. 2017); similar parameters $(P A=349 \pm 5$ and $i=56 \pm 5$ ) were obtained by Wu et al. (2017). In addition, when we assume that the circumprimary disk seen by ALMA is corotating with GQ Lup B, that is, is describing a counterclockwise orbit (see Schwarz et al. 2016), the near side also is the SW side in this case. This comparison suggests that the two disks have a similar but not identical orientation, being disaligned by $38.1 \pm 3.4$ degree.

\section{Is there an outflow from GQ Lup C?}

Astrometric calibration of the HST images was obtained using the position, proper motion, and parallax of GQ Lup A, C, and of two background stars (sources id. 60115227576375 61216 and 6011522757637551616$)$ measured by Gaia DR2 (Gaia Collaboration et al. 2016, 2018) as references. In this way, we obtained a plate scale of $0.03962 \operatorname{arcsec}$ pixel $^{-1}$ (very close to the nominal value of 0.0395 arcsec pixel ${ }^{-1}$ given in the WFC3 handbook ${ }^{5}$ and a true-north correction of 132.153 degree.

We then measured the position of GQ Lup $C$ in the individual bands with respect to the two reference background stars and between the two reference stars themselves. We found that the positions in most bands are essentially identical with each other, with an rms scatter of about 6 mas. However, the position of GQ Lup $\mathrm{C}$ measured on the image obtained with the $F 656 N$ filter (that is, at $\mathrm{H} \alpha$ ) is offset with respect to the average of that obtained at the remaining wavelengths by about 13 mas, at $P A=306 \pm 24$ degree measured on sky. While this result has a significance at only slightly more than twice the error bar, it suggests that $\mathrm{H} \alpha$ is emitted from a region that is not the same as for the remaining wavelengths. This also agrees with the lower value of the FWHM of the GQ Lup C image (112 mas) obtained with $F 656 N$ with respect an average value of 131 mas (with an rms of 10 mas) for the remaining wavelengths.

We propose that most of the $\mathrm{H} \alpha$ emission may be attributed to an outflow from GQ Lup C. The outflow is expected to be seen projected along the minor axis of the disk image on the far side, that is, roughly at $P A=45$ degree. This implies that the $\mathrm{H} \alpha$ emission seems to be shifted at almost right angle, but slightly in the opposite direction. This last feature might be explained as follows: while the direct stellar light is heavily absorbed by the disk and we see it largely as light scattered by the disk, that is, at a position substantially offset with respect to the real position of the star along the minor axis of the disk, the outflow is not significantly obscured. This agrees well with the discussion in Paper I. Variability of the emission is consistent with the small size of the outflow $(\sim 0.1 \mathrm{arcsec})$ and with the quite long time that has elapsed between HST and X-shooter observations (seven years).

\section{Discussion and conclusions}

The main result of this Letter is the detection of the disk around GQ Lup C both from the excess in the WISE W1 and W2 bands

https://hst-docs.stsci.edu/display/WFC3IHB 
and from scattered light seen in the HST images. The near IR excess reveals the warm part of the disk, whose emission is matched by a blackbody with a temperature of $800 \mathrm{~K}$. We note that this temperature is lower than that of dust sublimation, suggesting that the disk is truncated, probably by magnetic fields at the stellar corotation radius. This is not well determined because we can only estimate the stellar rotation period from the values of the stellar radius and of $V \sin i$ determined in Paper I, and using the inclination $i$ we obtained for the disk. The corresponding period is $2.7 \mathrm{~d}$, but it is largely uncertain because of the huge error bar of $V \sin i$. On the other hand, the inner disk radius is also uncertain because the value of the albedo is not well determined. However, an inner disk radius of $0.20 \mathrm{au}$, corresponding to a period of $2.7 \mathrm{~d}$ for the stellar mass $\left(0.15 M_{\odot}\right)$ determined in Paper I, is obtained assuming an albedo of 0.3 .

The scattered light image provides an estimate of the disk radius of $13.2 \pm 0.8 \mathrm{au}$. This is smaller than all disk radii obtained in the ALMA survey of disks in Lupus (Ansdell et al. 2016, 2018), but this is due to the resolution of the ALMA images used in that survey. There are examples of disks as small as this in GQ Lup C that have been resolved by ALMA (see, e.g., Facchini et al. 2019). On the other hand, this same survey suggests a correlation between disk radii and dust masses that is roughly described by the power law $M_{\mathrm{dust}} / M_{\oplus} \sim$ $0.044\left(R_{\text {dust }} / \mathrm{au}\right)^{1.5}$. While this relation is based on submm observations, which can provide very different images of disks with respect to scattered light, we note that if we were to use the disk radius determined from the HST data, we would obtain a dust mass of $2.1 M_{\oplus}$; this is a quite typical value for disks around low-mass stars in Lupus (Ansdell et al. 2016, 2018). The disk of GQ Lup C therefore does not appear to be anomalous for the Lupus association, and we expect that the corresponding submm flux would have been detected by that survey, although the resolution of the disk would have required a longer baseline.

We finally note that the orientation of the major axis of the disks of GQ Lup A and C agrees fairly well with the main axis of the filament that is visible in Herschel images (Rygl et al. 2013), while that of the minor axis agrees with that of the magnetic field in nearby regions (Rizzo et al. 1998; Matthews et al. 2014).

Acknowledgements. R.G., D.M., S.D. and V.D. acknowledge support from the "Progetti Premiali" funding scheme of the Italian Ministry of Education, University, and Research. This work has been supported by the project PRIN-INAF 2016 The Cradle of Life - GENESIS-SKA (General Conditions in Early Planetary Systems for the rise of life with SKA). E.R. is supported by the European Union's Horizon 2020 research and innovation programme under the Marie Skłodowska-Curie grant agreement No 664931. This work has made use of data from the European Space Agency (ESA) mission Gaia (https://www . cosmos.esa.int/gaia), processed by the Gaia Data Processing and Analysis Consortium (DPAC, https://www. cosmos.esa.int/web/gaia/dpac/ consortium). Funding for the DPAC has been provided by national institutions, in particular the institutions participating in the Gaia Multilateral Agreement.

\section{References}

Alcalá, J. M., Majidi, F. Z., Desidera, S., et al. 2020, A\&A, 635, L1

Allard, F., Homeier, D., \& Freytag, B. 2012, Phil. Trans. R. Soc. London Ser. A, 370,2765

Ansdell, M., Williams, J. P., van der Marel, N., et al. 2016, ApJ, 828, 46

Ansdell, M., Williams, J. P., Trapman, L., et al. 2018, ApJ, 859, 21

Bouvier, J., Alencar, S.H.P., Harries, T.J., Johns-Krull, C.M., \& Romanova, M.M. 2007, Protostars and Planets V, eds. B. Reipurth, D. Jewitt, \& K. Keil, 479

Facchini, S., van Dishoeck, E. F., Manara, C. F., et al. 2019, A\&A, 626, L2

Frasca, A., Montes, D., Alcalà, J. M., Klutsch, A., \& Guillout, P. 2018, Acta Astron., 68, 403
Gaia Collaboration (Prusti, T., et al.) 2016, A\&A, 595, A1 Gaia Collaboration (Brown, A. G. A., et al.) 2018, A\&A, 616, A1 Ginski, C., Stolker, T., Pinilla, P., et al. 2016, A\&A, 595, A112 Lazzoni, C., Zurlo, A., Desidera, S., \& Mesa, D. 2020, A\&A, submitted MacGregor, M. A., Wilner, D. J., Czekala, I., et al. 2017, ApJ, 835, 17 Matthews, T. G., Ade, P. A. R., Angilè, F. E., et al. 2014, ApJ, 784, 116 Merín, B., Jørgensen, J., Spezzi, L., et al. 2008, ApJS, 177, 551 Rizzo, J. R., Morras, R., \& Arnal, E. M. 1998, MNRAS, 300, 497 Ryg1, K. L. J., Benedettini, M., Schisano, E., et al. 2013, A\&A, 549, L1 Schwarz, H., Ginski, C., de Kok, R. J., et al. 2016, A\&A, 593, A74 Sissa, E., Gratton, R., Garufi, A., et al. 2018, A\&A, 619, A160 Skrutskie, M. F., Cutri, R. M., Stiening, R., et al. 2006, AJ, 131, 1163 Wright, E. L., Eisenhardt, P. R. M., Mainzer, A. K., et al. 2010, AJ, 140, 1868 Wu, Y.-L., Sheehan, P. D., Males, J. R., et al. 2017, ApJ, 836, 223

\section{Appendix A: Method for subtracting the PSF}

The method applied in Sect. 4 to image the disk around GQ Lup C after subtracting a model PSF is described in detail in Lazzoni et al. (2020). Here, we summarize two main caveats that are to be considered when this technique is used. The first regards the model that is used because we fit the parameters of a Gaussian profile for the central peak and damped correction for the Airy diffraction pattern (the model PSF of the nearby star) to a PSF that has a different shape (GQ Lup C), given by the presence of the disk. For this reason, the optimized residuals will show over-bright areas surrounded by over-subtracted areas to balance the incongruous models. Thus, one of the most difficult parameters to estimate is the flux of the source, whereas the geometry of the disk should be less affected, especially for disks that are centered around the star. In cases like the one presented in this paper, where the disk appears to be slightly miscentered, the derived inclination and width are to be considered as a first estimate only.

The second caveat instead concerns the differences between the PSF of the object and the PSF of the model due to the instruments itself. Even if the two PSFs are taken at the same time (thus in the same conditions), distortions may emerge from a different position in the field of view. However, because the two sources are quite close on the detector, we can neglect these effects for this case.

\section{Appendix B: Table}

Table B.1. Data for the SED of GQ Lup C.

\begin{tabular}{lccc}
\hline \hline Band & $\begin{array}{c}\text { Wavel. } \\
(\mu \mathrm{m})\end{array}$ & $\begin{array}{c}\text { Magnitude } \\
(\mathrm{mag})\end{array}$ & $\begin{array}{c}\text { Flux } \\
\mathrm{erg} \mathrm{cm}^{-2} \mathrm{~s}^{-1} \mathrm{~nm}^{-1}\end{array}$ \\
\hline HST-F336W & 0.3355 & $21.631 \pm 0.046$ & $(7.21 \pm 0.30) \mathrm{E}-17$ \\
HST-F390W & 0.3921 & $22.258 \pm 0.108$ & $(7.23 \pm 0.68) \mathrm{E}-17$ \\
HST-F475W & 0.4771 & $21.052 \pm 0.018$ & $(1.97 \pm 0.03) \mathrm{E}-16$ \\
HST-F555W & 0.5305 & $20.362 \pm 0.018$ & $(2.82 \pm 0.05) \mathrm{E}-16$ \\
HST-F625W & 0.6241 & $19.106 \pm 0.017$ & $(5.52 \pm 0.09) \mathrm{E}-16$ \\
HST-F656N & 0.6561 & $16.086 \pm 0.015$ & $(5.24 \pm 0.07) \mathrm{E}-16$ \\
HST-F673N & 0.6766 & $18.992 \pm 0.020$ & $(4.80 \pm 0.09) \mathrm{E}-16$ \\
HST-F775W & 0.7651 & $17.487 \pm 0.011$ & $(1.32 \pm 0.01) \mathrm{E}-15$ \\
HST-F850LP & 0.9187 & $16.366 \pm 0.012$ & $(2.25 \pm 0.02) \mathrm{E}-15$ \\
2MASS- $J$ & 1.235 & $14.849 \pm 0.052$ & $(3.60 \pm 0.17) \mathrm{E}-15$ \\
2MASS- $H$ & 1.662 & $14.083 \pm 0.048$ & $(2.64 \pm 0.11) \mathrm{E}-15$ \\
2MASS- $K$ & 2.159 & $13.818 \pm 0.049$ & $(1.27 \pm 0.06) \mathrm{E}-15$ \\
WISE-W1 & 3.353 & $12.60 \pm 0.38$ & $(7.47 \pm 2.21) \mathrm{E}-16$ \\
WISE-W2 & 4.603 & $12.20 \pm 0.40$ & $(3.17 \pm 0.98) \mathrm{E}-16$ \\
WISE-W3 & 12 & $<10.638$ & $<3.62 \mathrm{E}-17$ \\
\hline
\end{tabular}

